\title{
Isolated central nervous system leukemiarelapse presenting as Guillain-Barré-like syndrome:A case report
}

\begin{abstract}
We report the clinical picture, management and outcome of a 14-year-old boy treated for acute lymphoblastic leukemia (ALL) in whom isolated central nervous system relapse manifested by a Guillain-Barré-like syndrome (GBS). While on maintenance chemotherapy for his first CNS-relapse, he developed acute progressive lower motor neuron right facial palsy and marked hypotonia and areflexia of both lower limbs with otherwise no systemic manifestations. Vincristine-induced neuropathy (VIN) was suspected but his electrophysiological evaluation revealed a proximal demyelinating polyradiculoneuropathy pattern suggestive of GBS, but his cerebrospinal fluid analysis showed increased protein and infiltration by $95 \%$ leukemic blast cells. Immunoglobulins and chemotherapy were initiated. Although GBS in children with ALL is very rare, differentiating it from VIN by electrophysiological studies and excluding CNS relapse is crucial to determine the proper treatment and ensure a better clinical outcome.
\end{abstract}

Keywords: guillain-Barré syndrome, acute leukemia, relapse, neuropathy
Volume 8 Issue 3 - 2020

\author{
Marwa Abd El-Maksoud, ' Yasmine El Chazli,' \\ Yasser Wali ${ }^{2,3}$ \\ 'Department of Pediatrics, Neurology unit, Faculty of Medicine, \\ Alexandria University, Egypt \\ ${ }^{2}$ Department of Pediatrics, Hematology/Oncology unit, Faculty \\ of Medicine, Alexandria University, Egypt \\ ${ }^{3}$ Child Health Department, Sultan Qaboos University Hospital, \\ Muscat, Oman
}

Correspondence: Marwa Abd El-Maksoud, Department of Pediatrics, Neurology unit, Faculty of Medicine, Alexandria University, Egypt, Email mabdelmaksoudI6@alexmed.edu.eg

Received: June 07, 2020 | Published: June 23, 2020
Abbreviations: GBS, guillain barre syndrome; CNS, central nervous system; VIN, vincristine-induced neuropathy; LMN, lower motor neuron; CSF, cerebrospinal fluid

\section{Introduction}

Guillain Barre Syndrome (GBS), also known as acute acquired polyradiculoneuropathy, presents with progressive ascending muscle weakness sometimes progressing to complete paralysis. Although the exact etiology is still unknown, an immune-mediated mechanism is strongly suggested. Its incidence widely ranges between 0.4 and 4 cases per 100.000 people per year. ${ }^{1}$ Involvement of the central nervous system (CNS) in acute lymphoblastic leukemia (ALL), the most common childhood cancer, is a major clinical problem. Neurologic symptoms associated with CNS leukemia include headaches, nausea and vomiting, lethargy, and irritability and are usually due to direct infiltration by malignant cells, complications by a CNS infection or as a result of treatment adverse effects. ${ }^{2}$ The most frequently recorded acute treatment-induced neurological sequelae are methotrexateinduced leukoencephalopathy, vincristine-induced neuropathy (VIN) and L-asparaginase-induced strokes. ${ }^{2,3}$ We hereby report the case of a boy in whom leukemic relapse manifested by the picture of GBS.

\section{Case description}

A 14-year-old boy diagnosed as B-ALL at the age of 9 years in January 2013. His cerebrospinal fluid (CSF) analysis was free at diagnosis with no blast cells. He was treated according to the highrisk standard-arm of the modified children cancer group (CCG) 1961 protocol. ${ }^{4} \mathrm{He}$ achieved complete remission after the induction phase and received consequent cycles of chemotherapy punctuated with recurrent episodes of bone marrow suppression and hospital admissions for febrile neutropenia. After two years of maintenance therapy, in December 2015, he developed isolated CNS relapse presenting by headache and evidenced by the presence of blasts on his
CSF, while his bone marrow was still in remission. He was shifted to the isolated CNS relapse protocol adopted from the pediatric oncology group (POG) 9412. ${ }^{5} \mathrm{He}$ responded well to remission induction, went into consolidation and intensification phases, and received cranial irradiation. While still on maintenance chemotherapy, in January 2018, he complained of acute onset of mouth deviation, inability to close his eyelid, and difficulty walking. Physical examination revealed acute progressive lower motor neuron (LMN) right facial palsy and marked hypotonia and areflexia of both lower limbs with normal upper limbs and otherwise no systemic manifestations. The MRI brain and orbit were free. An electrophysiological evaluation revealed a proximal demyelinating polyradiculoneuropathy pattern suggestive of GBS. The motor conduction was tested bilaterally and showed delayed motor conduction velocity, decreased amplitude with poor $\mathrm{F}$ waves for all tested peripheral nerves on both lower limbs. The right facial nerve showed a poor response compared to the left side. The sensory nerve action showed only a poor response of superficial peroneal nerve with normal somatosensory evoked potentials on both upper and lower limbs, suggesting a peripheral rather than a central lesion. The $\mathrm{H}$ reflex was poor with absent $\mathrm{F}$ waves on both sides, suggesting an acute demyelinating process. Fundus examination showed bilateral papilledema, right sided retinal hemorrhage, and excaudate. Urgent brain CT was unremarkable. His magnetic resonance imaging of the brain and orbit did not show any evidence of leukemic infiltration. Blood investigations revealed leukopenia (2930 cell/cmm $\mu \mathrm{L})$, borderline platelet count $(153.000 \mathrm{c} / \mathrm{cmm} \mu \mathrm{L})$, and hemoglobin $10.7 \mathrm{~g} / \mathrm{dL}$. His CSF analysis showed protein of $132 \mathrm{mg} /$ $\mathrm{dL}$, neutrophils $25 \mathrm{cell} / \mathrm{cmm} \mu \mathrm{L}$, lymphocytes 2705 cell/cmm $\mu \mathrm{L}$ with $95 \%$ blast cells, while his bone marrow examination was negative for leukemic infiltration. Intravenous immunoglobulin (IVIG) was initiated at $(0.4 \mathrm{mg} / \mathrm{kg} /$ dose for 5 five days $)$, but he actually received only 3 three doses as he developed a severe anaphylactic reaction during the third infusion. Re-induction chemotherapy for isolated CNS relapse was concomitantly initiated using dexamethasone, 
vincristine, L-asparaginase, mitoxantrone, and intrathecal therapy. ${ }^{6}$ The child improved gradually, with the recovery of facial palsy within 2 two weeks and complete recovery of walking within 2 two months.

\section{Discussion}

This report describes a 14-year-old boy with a second isolated CNS relapse, which presented by acute progressive ascending paresis of both lower limbs with right-sided facial palsy while on maintenance therapy for B-ALL first CNS relapse. The differential diagnosis at presentation with peripheral neuropathy was VIN or GBS. Nerve conduction velocity examination after the onset of neurologic symptoms is mandatory, as the absent $\mathrm{F}$ waves indicate a proximal nerve conduction block caused by an acute demyelinating process allowing us to rule out acute VIN.

Peripheral neuropathy in the form of sensory-motor axonal polyneuropathy is a common adverse effect of vincristine that manifests in $30 \%$ to $50 \%$ of treated patients and is sometimes severe enough to cause complete paralysis . ${ }^{7}$ Whereas VIN is considered a relatively common cause of peripheral neuropathy in children with ALL receiving chemotherapy, ${ }^{8}$ only a few published reports describe acute inflammatory demyelinating polyradiculoneuropathy (AIDP) in these patients. Nine patients were reported so far in the literature, including; six children who developed GBS during induction; ${ }^{7,9-11}$ two were in remission, 7,12 and only one child presented with GBS as the initial presentation of underlying ALL. ${ }^{13}$

Although the clinical and electrophysiological studies of the presented patient were consistent with the AIDP subtype of GBS, a CSF analysis, which is mandatory not only to fulfill "“"Brighton diagnostic criteria" " for GBS but also because of the possibility of CNS-relapse in a known patient with ALL. ${ }^{14}$ It revealed the presence of blast cells indicating leukemic infiltration due to CNS relapse. The overlap of the GBS and CNS relapse might explain the clinical improvement of the child after the reinduction chemotherapy and IVIG.

Guillain-Barré syndrome is a common cause of acute flaccid paralysis and is strongly believed to be an autoimmune-mediated disease mostly triggered by infections. Molecular mimicry between microbial and nerve antigens has a major role, especially with Campylobacter jejuni infection, although GBS occurs in less than 1 in 1000 infected people. ${ }^{15}$ GBS had also been reported in association with various hematologic conditions, including Hodgkin and nonHodgkin lymphoma and ALL in adults. ${ }^{16-18}$

The pathophysiologic basis for AIDP in children with ALL remains unclear. Several explanations for its pathogenesis have been proposed. Immunosuppression secondary to intensive chemotherapy could pave the way to infectious triggers of AIDP. ${ }^{12}$ Neoplasms may also trigger AIDP in a manner similar to some viral infections. ${ }^{19}$ However, the exact mechanism, including the microbial or neoplastic and host factors, by which the immune response is shifted towards unwanted autoreactivity, is still not well understood. ${ }^{20} \mathrm{~A}$ paraneoplastic demyelinating sensorimotor neuropathy has been also suggested. ${ }^{13}$

In conclusion, although GBS in children with ALL is very rare, differentiating it from VIN by electrophysiological studies, and excluding underlying leukemia relapse, especially the isolated CNS type, is crucial to determine the proper diagnosis, treatment, and improve the clinical outcome.

\section{Acknowledgments}

The authors would like to thank the parents of the presented case for their approval of the publication of this report as well as the pediatric Hematology/Oncology team at Alexandria University Children's Hospital.

\section{Conflicts of interest}

The authors declare that they have no conflict of interest.

\section{Ethics approval}

Ethical approval was waived by the local Ethics Committee of University A in view of the retrospective nature of the study and all the procedures being performed were part of the routine care.

\section{Authors' contribution}

Marwa Abd El-Maksoud and Yasmine El Chazli were responsible for clinical diagnosis, management and follow up of the index case. The first draft of the manuscript was written by Marwa Abd El-Maksoud and Yasmine El Chazli and all authors commented on previous versions of the manuscript. All authors read and approved the final manuscript.

\section{References}

1. Sejvar JJ, Kohl KS, Gidudu J, et al. Guillain-Barre syndrome and Fisher syndrome: case definitions and guidelines for collection, analysis, and presentation of immunization safety data. Vaccine. 2011;29(3):599-612.

2. Hanmantgad S, Khakoo Y. 133 - System Cancer and the Central Nervous System Involvement. In: Swaiman KF, Ashwal S, Ferriero DM, et al. editors. Swaiman's Pediatric Neurology. 6th edn, Elsevier, 2017. pp. 1017-1020.

3. Smith MA, Seibel NL, Altekruse SF, et al. Outcomes for children and adolescents with cancer: challenges for the twenty-first century. Journal of Clinical Oncology. 2010;28(15):2625-2634.

4. Gaynon PS, Angiolillo AL, Carroll WL, et al. Long-term results of the children's cancer group studies for childhood acute lymphoblastic leukemia 1983-2002: a Children's Oncology Group Report. Leukemia. 2010;24(2):285-297.

5. Barredo JC, Devidas M, Lauer SJ, et al. Isolated CNS Relapse of Acute Lymphoblastic Leukemia Treated With Intensive Systemic Chemotherapy and Delayed CNS Radiation: A Pediatric Oncology Group Study. Journal of Clinical Oncology. 2006;24(19):3142-3149.

6. Parker C, Waters $\mathrm{R}$, Leighton $\mathrm{C}$, et al. Effect of mitoxantrone on outcome of children with first relapse of acute lymphoblastic leukaemia (ALL R3): an open-label randomised trial. Lancet (London, England.) 2010;376(10):2009-2017.

7. Brigo F, Balter R, Marradi P, et al. Vincristine-related neuropathy versus acute inflammatory demyelinating polyradiculoneuropathy in children with acute lymphoblastic leukemia. J Child Neurol. 2012;27(7):867-874.

8. Nazir H, Al Futaisi A, Mathew Zacharia, et al. Vincristine-induced neuropathy in pediatric patients with acute lymphoblastic leukemia in Oman: Frequent autonomic and more severe cranial nerve involvement. Pediatric blood \& cancer. 2017;64(12).

9. Rajeswari B, Krishnan S, Sarada C, et al. Guillain-Barre Syndrome with Acute Lymphoblastic Leukemia. Indian pediatrics. 2013;50(8):791-792.

10. Norman M, Elinder G, Finkel Y. Vincristine neuropathy and a GuillainBarre syndrome: a case with acute lymphatic leukemia and quadriparesis. Eur J Haematol. 1987;39(1):75-76. 
11. Bhushan B, Bhargava A, Kasundra GM, et al. Guillain-Barre syndrome in acute lymphoblastic leukemia: Causal or coincidental. J Pediatr Neurosci. 2015;10(1):64-66.

12. Aral YZ, Gursel T, Ozturk G, et al. Guillain-Barre syndrome in a child with acute lymphoblastic leukemia. Pediatr Hematol Oncol. 2001;18(5):343346 .

13. Chandar R, Seetharam S, Gopakumar KG, et al. Paraneoplastic Demyelinating Sensorimotor Neuropathy Delaying the Diagnosis of an Underlying Acute Lymphoblastic Leukemia in a Child. J Oncol Pract. 2018;14(10):629-631.

14. Roodbol J, de Wit M-CY, van den Berg B, et al. Diagnosis of GuillainBarré syndrome in children and validation of the Brighton criteria. $J$ Neurol. 2017;264(5):856-861.

15. Huizinga $\mathrm{R}$, van den Berg $\mathrm{B}$, van Rijs W, et al. Innate Immunity to Campylobacter jejuni in Guillain-Barre Syndrome. Annals of neurology. 2015;78(3):343-354.
16. Re D, Schwenk A, Hegener P, et al. Guillain-Barre syndrome in a patient with non-Hodgkin's lymphoma. Ann Onco. 2000;11(2):217-220.

17. Hughes CL, Yorio JT, Kovitz C, et al. Treatment decisions in a man with Hodgkin lymphoma and Guillian-Barré syndrome: a case report. $J$ Med Case Rep. 2014;8:455.

18. Vembu P, Al-Shubaili A, Al-Khuraibet A, et al. Guillain-Barré Syndrome in a Case of Acute Lymphoblastic Leukaemia. Med Princ Pract. 2003;12(4):272-275.

19. Moudgil SS, Riggs JE. Fulminant peripheral neuropathy with severe quadriparesis associated with vincristine therapy. Ann Pharmacother. 2000;34(10):1136-1138.

20. Willison HJ, Goodyear CS. Glycolipid antigens and autoantibodies in autoimmune neuropathies. Trends in immunology. 2013;34(9):453-459. 